\title{
(W)WILEY
}

\section{Two phases of replacement replenish the olfactory ensheathing cell population after injury in postnatal mice}

\begin{tabular}{|c|c|}
\hline Journal: & GLIA \\
\hline Manuscript ID: & GLIA-00135-2011.R3 \\
\hline Wiley - Manuscript type: & Original Research Article \\
\hline Date Submitted by the Author: & $\mathrm{n} / \mathrm{a}$ \\
\hline Complete List of Authors: & $\begin{array}{l}\text { Chehrehasa, Fatemeh; Griffith University, Eskitis Institute for Cell and } \\
\text { Molecular Therapies } \\
\text { Ekberg, Jenny; Griffith University, Eskitis Institute for Cell and Molecular } \\
\text { Therapies } \\
\text { Lineburg, Katie; Griffith University, Eskitis Institute for Cell and Molecular } \\
\text { Therapies } \\
\text { Amaya, Daniel; Griffith University, Eskitis Institute for Cell and Molecular } \\
\text { Therapies } \\
\text { Mackay-Sim, Alan; Griffith University, Eskitis Institute for Cell and } \\
\text { Molecular Therapies } \\
\text { St John, James; Griffith University, Eskitis Institute for Cell and Molecular } \\
\text { Therapies }\end{array}$ \\
\hline Key Words: & Regeneration, migration, axon, OEC, glia \\
\hline
\end{tabular}

SCHOLARONE ${ }^{\text {m }}$

Manuscripts 
Two phases of replacement replenish the olfactory ensheathing cell population after injury in postnatal mice

Fatemeh Chehrehasa ${ }^{1,2}$, Jenny A.K. Ekberg ${ }^{1}$, Katie Lineburg ${ }^{1,3}$, Daniel Amaya ${ }^{1}$, Alan Mackay-Sim ${ }^{1}$ and James A. St John ${ }^{1}$

${ }^{1}$ National Centre for Adult Stem Cell Research, Eskitis Institute for Cell and Molecular Therapies, Griffith University, 170 Kessels Rd, Nathan 4111, Brisbane, Queensland, Australia

${ }^{2}$ Current address: School of Medical Sciences, Queensland University of Technology, 2 George Street, Brisbane 4000, Queensland, Australia

${ }^{3}$ Current address: Clive Berghofer Cancer Research Centre, Queensland Institute of Medical Research, 300 Herston Road, Herston 4006, Brisbane, Queensland, Australia

Running title: Olfactory glia proliferate after injury

Number of words: Abstract 231; Introduction 555; Materials and methods 712; Results 1682; Discussion 1152; References 958; Legends 1446; Total word count: 6958.

Number of Figures: 9

Number of Tables: 0

Corresponding author:

Dr James St John

Eskitis Institute for Cell and Molecular Therapies

Griffith University

170 Kessels Rd

Nathan 4111

Brisbane

Australia

Phone +61-7-3735 3660

Fax +61-7-3735 4255

Email: j.stjohn@griffith.edu.au

Key words: regeneration, migration, glia, axon, OEC 


\begin{abstract}
Olfactory ensheathing cells (OECs) support the regeneration of olfactory sensory neurons throughout life, however it remains unclear how OECs respond to a major injury. We have examined the proliferation and migration of OECs following unilateral bulbectomy in postnatal mice. S100ß-DsRed and OMP-ZsGreen transgenic mice were used to visualise OECs and olfactory neurons, respectively, and we used the thymidine analogue ethynyl deoxyuridine (EdU) to identify cells that were proliferating at the time of administration. Following unilateral bulbectomy, there was an initial phase of OEC proliferation throughout the olfactory pathway with a peak of proliferation occurring 2-7 days after the injury. A second phase of proliferation also occurred in which precursors localised within the olfactory mucosa divided to replenish the OEC population. We then tracked the positions of OECs that had proliferated and found that there was a progressive increase in OECs in the cavity for at least 12-16 days after injury which could not be accounted for solely by local proliferation of OECs within the cavity. These results suggest that OECs migrated from the peripheral olfactory nerve to populate the mass of cells that filled cavity left by bulbectomy. Our results demonstrate that following injury to the olfactory nervous system, the OEC population is replenished by migration of cells that arise from both local proliferation of OECs throughout the olfactory nerve pathway as well as from precursor cells in the olfactory mucosa.
\end{abstract}




\section{INTRODUCTION}

The olfactory system has the unique ability of continuously replacing olfactory receptor neurons that are lost by normal turnover and by injury (Farbman and Squinto 1985; Graziadei and Graziadei 1979b; Mackay-Sim and Kittel 1991a; Mackay-Sim and Kittel 1991b). The growth and regeneration of olfactory sensory axons is partly attributable to the specialised glial cells of the olfactory system, the olfactory ensheathing cells (OECs) that form the fascicles through which the axons grow from the peripheral nervous system in to the central nervous system (Boyd et al. 2005; Chuah and Au 1991; Doucette 1984).

The role of OECs in the regeneration of the olfactory nerve has led to their application in spinal cord injury repair (Feron et al. 2005; Li et al. 2003; Li et al. 1997; Lu et al. 2001; Mackay-Sim et al. 2008; Ramon-Cueto and Nieto-Sampedro 1994; Ramon-Cueto et al. 1998). Several studies have now shown that OECs transplanted into the injured spinal cord have proliferated and migrated throughout the injury site (Boruch et al. 2001; Deng et al. 2006; Gorrie et al. 2010; Ramon-Cueto and Nieto-Sampedro 1994; Resnick et al. 2003) but there are experimental variations in neuro-regeneration. Functional outcomes may be improved in future by a better understanding of the response of OECs to injury. The goal of this study is to determine the OEC response to injury in its normal cellular milieu, as glial cells of the olfactory nerve. Following injury that causes death of primary olfactory neurons, stem cells generate new neurons that replenish the olfactory epithelium (Graziadei and Graziadei 1979a; Graziadei et al. 1978; Iwai et al. 2008; Jang et al. 2003; Leung et al. 2007). In contrast little is known about how OECs respond after injury to the olfactory nerve. By better understanding the proliferative and migratory responses of OECs within the olfactory system, it may be possible to improve the use of OECs in neural regeneration therapies. 
Chemical destruction of the olfactory epithelium or transection of the olfactory nerve leads to loss of the olfactory sensory neurons but has minimal effects on proliferation of OECs (Li et al. 2005; Williams et al. 2004) and low or negligible levels on OEC migration (Chuah et al. 1995; Li et al. 2005; Williams et al. 2004). Neither of these injuries causes significant loss of OECs, whereas removal of the olfactory bulb simultaneously removes a large proportion of OECs and leads to widespread loss of olfactory sensory neurons. After unilateral bulbectomy there is a large influx of OECs into the cavity left by the bulbectomy (Chehrehasa et al. 2008; Chehrehasa et al. 2010) but the location of the source of these OECs is not known. Do they arise from local proliferation? Do they migrate from more peripheral sources in the lamina propria? Do they arise from proliferation of precursor cells in the olfactory epithelium? To answer these questions we investigated the proliferation and migration of OECs within the nasal cavity and skull base following unilateral bulbectomy in postnatal pups. We found that bulbectomy stimulated an early transient proliferation throughout the olfactory nerve driving migration of OECs into the skull base cavity for up to 16 days. Additionally, we found evidence for a delayed generation of new OECs from a non-OEC cell population such as the basal stem cells of the olfactory epithelium (Leung et al. 2007; Mackay-Sim and Kittel 1991a). 


\section{MATERIALS AND METHODS}

\section{Animals}

We used animals of two transgenic mouse lines: OMP-ZsGreen mice, expressing ZsGreen in primary olfactory sensory neurons (Ekberg et al. 2011; Windus et al. 2010) and S100ßDsRed mice, expressing DsRed in OECs (Windus et al. 2007). For some experiments, the two lines were crossed. All procedures were carried out with the approval of the Griffith University Animal Ethics Committee under the guidelines of the National Health and Medical Research Council of Australia and the Australian Commonwealth Office of the Gene Technology Regulator.

\section{Surgical ablation of olfactory bulb (bulbectomy)}

The olfactory bulb was removed unilaterally at postnatal day (P) 4.5 as described previously (Chehrehasa et al. 2005). Three animals in each treatment group at each time point were used.

\section{EdU administration}

Animals that had undergone bulbectomy treatment, as well as unoperated control animals, were examined at different times after treatment $(2,5,7,10,14$ days). To determine cell proliferation at each time point, the animals were given intraperitoneal injections of EdU (5ethyl-2'-deoxyuridine, Invitrogen USA, $50 \mathrm{mg} / \mathrm{kg}$ body weight in phosphate buffered saline (PBS) (pH 7.35) and sacrificed $4 \mathrm{~h}$ later. The second group of animals received a single EdU injection at 5 days after bulbectomy and harvested at 0,3, 7, and 11 days after EdU injection. 


\section{Tissue fixation and preparation}

Mice were killed by cervical dislocation and the heads fixed by immersion in $4 \%$ paraformaldehyde in PBS at room temp for 2-4 h depending on age.Heads from animals older than P10 were decalcified in $20 \%$ disodium ethylene diaminetetraacetic acid (EDTA) in PBS. The heads were then placed in an embedding matrix (O.C.T. compound, Miles Scientific, Naperville, IL) and snap frozen in iso-pentane cooled with liquid nitrogen. Cryostat sections $(30 \mu \mathrm{m})$ were cut, mounted on to slides and stored at $-20{ }^{\circ} \mathrm{C}$ before processing for EdU chemistry and immunohistochemistry.

\section{EdU chemistry}

EdU labelling was visualised using the Click-iT ${ }^{\mathrm{TM}}$ EdU Cell Proliferation Assay Kit (Cat\# C35002, Invitrogen).

\section{Immunohistochemistry}

Immunohistochemistry was performed as previously described (Chehrehasa et al. 2008). Antibodies used were polyclonal rabbit anti-S100 (1:400; Dako, Denmark), polyclonal rabbit anti-brain lipid binding protein (BLBP; 1:1000; Millipore Corporation, Billerica, MA), polyclonal rabbit anti-Iba1 (1:1000; Wako, Japan) and secondary antibodies Alexa Fluor 647 or Alexa Fluor 48 (1:200; Invitrogen). Cell nuclei were stained with 4',6-diamidino-2phenylindole.

\section{Image capture and image preparation}

Images were captured using an Axio Imager Z1 epi-fluorescence microscope with Apotome and an Axiocam Mrm camera (Carl Zeiss, Germany). Serial optical sections of up to $30 \mu \mathrm{m}$ in depth were captured using AxioVision software and projected to provide two-dimensional 
maximum brightness images. For quantification, the serial optical sections of each image were used to verify the co-localisation of EdU-labelling with OECs. Figures were compiled in Adobe Photoshop CS5 and Adobe Illustrator CS5 (Adobe Systems Incorporated).

\section{Quantification of OECs}

For each time point, three animals in each treatment group were examined. A total count of cells in each animal was obtained by using at least three sections per animal, with the total number of cells counted being at least 30-90 for each animal. Regions of interest (200 x 150 $\mu \mathrm{m}^{2}$ ) from specific areas of the olfactory epithelium, cribriform plate and olfactory bulb were selected as indicated in Figs 3A, 4A and 5A. The positions of these areas of interest (AOI) were kept constant between sections and animals so that similar regions of interest amongst the animals were examined. We counted the number of OECs as well as the number of OECs that were co-labelled with EdU. For each of the three regions (lamina propria, cribriform plate, nerve fibre layer), we determined a combined total single $\mathrm{n}$ value by adding together the cell counts from the same areas of interest across the different sections. Thus for each animal we had a single $\mathrm{n}$ value for the percentage of Edu-labelled OECs in the lamina propria, in the cribriform plate and in the nerve fibre layer/cavity of the olfactory bulb. For statistical tests and graphical presentation, all groups had $\mathrm{n}$ values of 3 . Statistical significance was first assessed using a two-way ANOVA; if $p$ values of less than 0.05 were returned for the groups of interest we then performed Tukey's post hoc test to determine where the significance lay within the groups with significance being accepted with a $p$ value of less than 0.05 . 


\section{RESULTS}

\section{Removal of the olfactory bulb induced proliferation of OECs}

To determine whether the proliferation and migration of OECs can be stimulated by a major injury in which the OEC population within the central nervous system (central OECs) is directly perturbed, we performed unilateral bulbectomy in postnatal mice. Unilateral ablation of an olfactory bulb ("bulbectomy") removes the central nervous tissue, the entire olfactory nerve fibre layer surrounding it, and the olfactory nerve bundles at the skull base at the cribriform plate, leaving a cavity (Fig. 1A). The contralateral olfactory bulb is relatively unaffected (Fig. 1A). Postnatal animals were chosen in preference to adults as the regeneration of olfactory neurons is more rapid and uniform (Chehrehasa et al. 2010; Hendricks et al. 1994). To easily visualise OECs and olfactory sensory axons we used transgenic fluorescent reporter mice in which OECs fluoresce red and olfactory neurons fluoresce green (S100ß-DsRed mice crossed with OMP-ZsGreen mice; Ekberg et al. 2011; Windus et al. 2007; Windus et al. 2010). In unilaterally bulbectomised mice, the resulting damage of the sensory axons led to degeneration of the neurons in the ipsilateral olfactory epithelium, which became thinner and had fewer ZsGreen-positive olfactory neurons (left nasal cavity, NC-1, Fig. 1A) compared to the contralateral unoperated side (NC-r, Fig. 1A). Within two days OECs and sensory axons had commenced infiltrating the injury site and formed a plexus in the ventral region of the cavity (Fig. 1D).

To determine whether OECs proliferated in response to the bulbectomy, proliferating cells were labelled by uptake of EdU at Days 2-14 after bulbectomy. We administered EdU at Days 2-14 after bulbectomy and sacrificed the animals $4 \mathrm{~h}$ after injection to provide a snapshot of the cells that were proliferating at each time point and to determine their location 
within the olfactory nerve. At Day 2, numerous cell types were positive for EdU in the olfactory mucosa, nasal septum, cartilage and in the skull base cavity (Fig. 1A) which confirmed that uptake of EdU was successful in all regions. In S100ß-DsRed transgenic mice, OECs are easily identified by their expression of DsRed, their morphology and their association with axons (see Fig. 1-2). Based on these characteristics, numerous OECs were clearly labelled by EdU in the lamina propria underlying the olfactory epithelium (arrow, Fig.1B-C) and in the skull base cavity (arrow, Fig. 1D-E). We further confirmed the identity of OECs by immunostaining with antibodies to two proteins that are markers of OECs: brain lipid binding protein (BLBP) and S100ß (Fig. 2C and 2H) (Murdoch and Roskams 2008). Using the three markers of OECs (S100ß, BLBP, DsRed) as well as morphology we confirmed that OECs that had proliferated at the time of EdU exposure were present in (1) the lamina propria underlying the olfactory epithelium along the nasal septum, (2) the large olfactory nerve bundles projecting through the cribriform plate at the skull base and (3) the nerve plexus formed within the skull base cavity left after bulbectomy (arrow, Fig. 1B-E; arrows in Fig. 2D, I). As expected, not all OECs were proliferating at the time of EdU exposure and thus many were EdU-negative (arrowhead, Fig. 1B-E; unfilled arrowheads in Fig. 2D, I). A subpopulation of macrophages which expressed Iba1 also expressed DsRed (Fig. 2K), but their distinct morphology and intense DsRed expression (Fig. 2L) made them readily identifiable and these cells were not included in the quantification of OECs.

\section{OECs proliferated throughout the olfactory nerve}

To determine the proportion of OECs that had proliferated we examined OECs in the three regions of the olfactory nerve: (1) the lamina propria (Fig. 3A), (2) the cribriform plate (Fig. 4A), and (3) the nerve plexus formed within the skull base cavity, and the nerve fibre layer of the contralateral olfactory bulb or unoperated control animals (Fig. 5A). After bulbectomy 
numerous EdU-positive OECs were present in all three regions at all periods examined. On the contralateral, unoperated side, there were fewer EdU-labelled OECs and in unoperated animals even fewer EdU-labelled OECs were seen (Figs. 3-5).

In the lamina propria ipsilateral to the bulbectomy, OECs underwent considerable proliferation (Fig. 3A), with significantly more EdU-labelled OECs compared to unoperated controls at Day 2-10 (Fig. 6A, two-way ANOVA $\mathrm{p}<0.0001$ for differences between treatments; $\mathrm{p}<0.05$ post hoc test for individual comparisons). The peak of proliferation occurred at Day 5-7 with EdU-labelled OECs comprising 10-11.5\% of the total population. OECs also proliferated in the lamina propria contralateral to the bulbectomy with $5-7 \%$ of OECs being EdU-positive (Fig. 6A). In contrast, there were many fewer EdU-labelled OECs in control, unoperated animals (1-2\% at Day 5-7, Fig. 6A). By Day 14, the proliferation of OECs in bulbectomised animals was similar to unoperated controls and was less than $2 \%$. In the region close to the cribriform plate ipsilateral to the bulbectomy, there was increased OEC proliferation (Fig. 4A) reaching a peak at Day 5 and with significantly more EdUlabelled OECs compared to the unoperated side and in control animals (Fig. 6B, two-way ANOVA $\mathrm{p}<0.0001$ for differences between treatments; $\mathrm{p}<0.05$ post hoc test for individual comparisons).

In the cavity left by bulbectomy, OEC proliferation was already at a peak at Day 2 and remained high at Day 5, then was reduced at Day 7 and Day 10 (Fig. 5A-C; Fig. 6C, two-way ANOVA $\mathrm{p}<0.0001$ for differences between treatments; $\mathrm{p}<0.05$ post hoc test for individual comparisons). On the unoperated contralateral side, there was a small, but significant $(\mathrm{p}<0.05)$ increase in EdU-positive OECs in all regions $($ Fig. 5D-F) reaching a maximum at 
Day 5 (Fig 6). In control animals, only a few EdU-positive OECs were detected (Figs. 5 and $6)$.

These results demonstrate that OECs proliferated in all regions of the olfactory nerve with high levels of proliferation occurring for 2-7 days after bulbectomy.

\section{Migration of OECs after bulbectomy}

Although maximal OEC proliferation within the bulbar cavity occurred at Day 2-5 after bulbectomy, the skull base cavity continued to fill with OECs for at least $14 \mathrm{~d}$ (Fig. 7A-C). With low rates of OEC proliferation during the later period (Fig. 6C) the increase in OECs in the skull base cavity must be due in part to migration of OECs from more peripheral regions of the olfactory nerve. To follow this migration we "birth-dated" OECs with EdU at Day 5, the maximal period of proliferation in the lamina propria and cribriform plate regions, and looked for EdU-labelled OECs in the different regions over the next 11 days. In all regions there was a delayed and significant increase in the numbers of labelled OECs at $7 \mathrm{~d}$ (lamina propria, cribriform plates) or 11d (skull base cavity) after birth-dating (Fig. 7D-F, two-way ANOVA $\mathrm{p}<0.001$ for differences between treatments; $\mathrm{p}<0.01$ post hoc test for individual comparisons). At these periods after bulbectomy (Day 12 and Day 16) the levels of OEC proliferation were very low in these regions in the snap-shot EdU labelling (Fig. 6) indicating that the increased numbers of EdU-labelled OECs were not due to additional cycles of proliferation. At Day 12 (seven days after EdU injection) there was a significantly greater percentage of EdU-positive OECs in the lamina propria $(\mathrm{p}<0.01$, Fig. 7D) and cribriform plate (p<0.01, Fig. 7E) in comparison to Day 5 (day of EdU injection) with $14-15 \%$ of OECs being EdU-positive. Thereafter, the proportion of EdU-positive OECs decreased. In the skull base cavity there was a significant increase at Day 16 (11 days after EdU injection) with just 
over $14 \%$ of OECs being EdU-positive (Fig. 7F). For comparison, in the snap-shot labelling only $1 \%$ of OECs were proliferating in this region at this time (Fig. 6C). For ease of analysis and comparisons, we had used percentage of OECs for graphical display. However, we considered that the increase in percentage of EdU-positive OECs may be a result of death of OECs. We therefore quantified the number of OECs per unit area. While there was a slight decrease in the number of OECs over Days 8-16 (Fig. 7G; $p<0.05$ two-way ANOVA for differences between time points, $\mathrm{p}<0.05$ for Days 8-16 Tukey's post-hoc test), the decrease was observed for both the operated and contralateral unoperated sides and there was no significant difference between the operated and contralateral sides $(\mathrm{p}>0.05)$ and therefore does not account for the large increase in the percentage of EdU-positive OECs observed only in the operated side (Fig. 7D-F). As the analyses were performed in postnatal animals, the slight decrease in density may be due to developmental changes. Thus the increase in percentage of EdU-positive OECs only in the operated side cannot be assigned to cell death, but more likely was the result of migration of EdU-positive OECs into the different regions.

From where did the migrating OECs arise that can account for the peaks of EdU-labelled OECs at Day 12 and 16? While some may have migrated away from regions of higher density created by increased proliferation, such as close to the cribriform plate, there was no corresponding significant decrease in OECs in those regions in comparison to contralateral unoperated sides between Days 8-16 (Fig. 7G; p>0.05). Alternatively, we considered that OECs may have arisen, de novo, from stem cell populations within the olfactory mucosa, for example, basal cells of the olfactory epithelium (Leung et al. 2007; Mackay-Sim and Kittel 1991a). We therefore examined the occurrence of EdU-labelled cells and OECs in the olfactory mucosa. At Day 5 after bulbectomy, there were numerous EdU-labelled basal cells in the olfactory epithelium (Fig 8A) and by Day 8 after bulbectomy most of the EdU-labelled 
cells were now located in the neuronal layer of the epithelium (arrowhead, Fig. 8F, I). Over the same period, the numbers of EdU-positive OECs in the lamina propria increased (arrows, Fig. 8B, E, H). At Day 12 (7 d after EdU labelling) the proportion of EdU-positive cells in the lamina propria was $14 \%$, significantly greater than on the unoperated side (4\%, Fig 7D) and much higher than the proportion of OECs proliferating in the lamina propria at this time (Fig, 6A). Thus these results indicate that the increase in EdU-labelled OECs in the lamina propria coincided with the movement of EdU-labelled cells out of the basal layer of the olfactory epithelium. 


\section{DISCUSSION}

In the present study we show that OECs proliferate and migrate along the olfactory pathway after unilateral removal of the olfactory bulb. Bulbectomy stimulated OEC proliferation close to the site of lesion within the skull base cavity, and distally within the lamina propria. This transient stimulation was maximal at $2 \mathrm{~d}$ in the skull base and at $5 \mathrm{~d}$ more distally, with OEC proliferation reducing to control levels at $14 \mathrm{~d}$. Birth-dating of OECs proliferating at Day 5 demonstrated significant migration of newly formed OECs into the skull base cavity.

When unilateral bulbectomy was performed, all cells within the olfactory bulb including the OECs within the nerve fibre layer were ablated. Within two days after the surgery OECs had proliferated in all regions of the olfactory nerve including within the bulbar cavity where OECs had already infiltrated from the remnant of the olfactory nerve. The early infiltration of OECs into the cavity was consistent with our previous findings where we have shown that a plexus of OECs and axons forms in the cavity (Chehrehasa et al. 2010). The elevated levels of proliferation in our bulbectomy model demonstrate that the OEC population can dynamically respond to an injury. In other models of regeneration in which primary olfactory axons have been destroyed but the OEC population has not been directly ablated, there have been negligible levels of proliferation of OECs (Chuah et al. 1995; Li et al. 2005; Williams et al. 2004). In adult rats, zinc sulphate irrigation of the nasal cavity has been used to destroy the olfactory epithelium resulting in the death of primary olfactory neurons and their axons that project to the olfactory bulb. However, the OECs along the olfactory nerve that encase the axon fascicles were not directly affected by the destruction of the olfactory epithelium and proliferation of OECs was not detected (Williams et al. 2004). Similarly, in an olfactory nerve transection model in adult rats, there was reported to be no proliferation of OECs 
following the loss of the olfactory axons ( $\mathrm{Li}$ et al. 2005). Instead, it was proposed that the OECs provide continuous stable open channels through which the regenerating axons extend. While we used neonatal mice in our model of regeneration whereas the other models used adult rats, our results clearly show that OECs are able to proliferate under certain conditions. This suggests that the source of the signal that stimulates the proliferation of OECs is not originating from the dying sensory neurons as OEC proliferation in all models would have been detected. The removal of the olfactory bulb created considerable space into which cells could infiltrate and which could have been a stimulus for the proliferation. If the availability of space was the only stimulus necessary then it would have been expected that OECs would have proliferated only in the cribriform plate region which is in close proximity to the cavity. However, OECs within the more distal lamina propria strongly responded suggesting that a more generalised mechanism throughout the olfactory nerve must be operating to stimulate the proliferation in all regions. Interestingly, there was a small stimulation of OEC proliferation on the unoperated contralateral side. While great care was taken during the surgery, it is possible that the contralateral bulb was damaged. Alternatively, there may have been a generalised mechanism such as an inflammatory response or humoral signals throughout the olfactory nerve that stimulated proliferation.

After the initial phase of proliferation, the cavity continued to be filled by OECs over the next 2-3 weeks. However, the percentage of OECs that had proliferated within the cavity in the later stages was very low, which suggested that OECs had proliferated in other regions and then migrated into the bulbar cavity (Fig. 9). The EdU birth-dating experiment revealed that a second peak of EdU-labelled OECs appeared at Day 12 after bulbectomy. Then four days later, a peak of EdU-labelled OECs appeared in the bulbectomised cavity and thus we conclude that OECs migrated from the lamina propria into the cavity. The migration of the 
OECs towards the olfactory bulb in our model is similar to that reported previously in onemonth old mice that had been subjected to zinc sulphate destruction of the olfactory epithelium (Chuah et al. 1995). In those mice, OECs migrated towards the olfactory bulb in the seven-day period after treatment even though the OEC population was not directly affected. However, in adult rats others have reported that there was minimal or negligible migration of OECs following zinc sulphate irrigation (Williams et al. 2004) or olfactory nerve transection ( $\mathrm{Li}$ et al. 2005). Nevertheless in our bulbectomy model it is clear that numerous OECs migrated into the cavity to form the plexus and thus the ability of OECs to migrate within the olfactory system may depend on the age of the animal and conditions of the injury. In spinal cord injury repair models, transplanted OECs have been shown to migrate extensively within the injury site and along the spinal cord (Deng et al. 2006; Lankford et al. 2008). When considered in conjunction with our results, it is therefore likely that the migratory properties of OECs are strongly dependent on the local microenvironment in which the OECs are located.

The source of the second peak of OECs is likely to be precursor cells that did not express OEC markers at Day 5 when the EdU was applied. Following loss of the neurons within the olfactory epithelium, the stem cells within the basal layer proliferated to give rise to new neurons that migrated apically to repopulate the olfactory epithelium, consistent with previous findings (Iwai et al. 2008; Jang et al. 2003; Leung et al. 2007). Without having lineage tracing of the OECs it is not possible to be certain of the origin of the later appearing OECs, however the timing of the appearance of the later appearing OECs coincided with the reduction in EdU-labelled cells in the basal layer of the olfactory mucosa. It is also possible that the later appearing OECs arose from sources elsewhere within the olfactory nerve, for example OECs could have migrated to the lamina propria from other regions of the nerve. 
We consider that this is unlikely as we could not identify any source of EdU-labelled cells in other regions of the olfactory nerve that showed similar timing to the appearance of the later appearing OECs. Thus our results suggest that the later appearing OECs came from a source within the olfactory mucosa (Fig. 9). It has recently been shown that OEC precursors arise from the neural crest and migrate to the olfactory region where they then proliferate to populate the olfactory nerve (Barraud et al. 2010). It remains unknown how long the neural crest derived OEC precursors persist within the olfactory nerve, but our results now show that at least in neonatal animals OECs can be repopulated from local proliferation of OECs throughout the olfactory pathway as well as from precursor cells in the olfactory mucosa. 


\section{Acknowledgments}

This work was supported by grant from the National Health and Medical Research Council to J.S (grant number 511006), by funding to the National Centre for Adult Stem Cell Research from the Australian Government Department of Health and Aging to A.M.S, and by an Australian Research Council Postdoctoral Fellowship to J.E. 


\section{References}

Barraud P, Seferiadis AA, Tyson LD, Zwart MF, Szabo-Rogers HL, Ruhrberg C, Liu KJ, Baker CV. 2010. Neural crest origin of olfactory ensheathing glia. Proc Natl Acad Sci U S A 107(49):21040-21045.

Boruch AV, Conners JJ, Pipitone M, Deadwyler G, Storer PD, Devries GH, Jones KJ. 2001. Neurotrophic and migratory properties of an olfactory ensheathing cell line. Glia 33(3):225-229.

Boyd JG, Doucette R, Kawaja MD. 2005. Defining the role of olfactory ensheathing cells in facilitating axon remyelination following damage to the spinal cord. Faseb $\mathrm{J}$ 19(7):694-703.

Chehrehasa F, Key B, St John JA. 2008. The cell surface carbohydrate blood group A regulates the selective fasciculation of regenerating accessory olfactory axons. Brain Res 1203:32-38.

Chehrehasa F, St John J, Key B. 2005. The sorting behaviour of olfactory and vomeronasal axons during regeneration. J Mol Histol 36(6-7):427-436.

Chehrehasa F, Windus LC, Ekberg JA, Scott SE, Amaya D, Mackay-Sim A, St John JA. 2010. Olfactory glia enhance neonatal axon regeneration. Mol Cell Neurosci 45(3):277-288.

Chuah MI, Au C. 1991. Olfactory Schwann cells are derived from precursor cells in the olfactory epithelium. J Neurosci Res 29(2):172-180.

Chuah MI, Tennent R, Jacobs I. 1995. Response of olfactory Schwann cells to intranasal zinc sulfate irrigation. J Neurosci Res 42(4):470-478.

Deng C, Gorrie C, Hayward I, Elston B, Venn M, Mackay-Sim A, Waite P. 2006. Survival and migration of human and rat olfactory ensheathing cells in intact and injured spinal cord. J Neurosci Res 83(7):1201-1212.

Doucette JR. 1984. The glial cells in the nerve fiber layer of the rat olfactory bulb. Anat Rec 210(2):385-391.

Ekberg JA, Amaya D, Chehrehasa F, Lineburg K, Claxton C, Windus LC, Key B, MackaySim A, St John JA. 2011. OMP-ZsGreen fluorescent protein transgenic mice for visualisation of olfactory sensory neurons in vivo and in vitro. $\mathrm{J}$ Neurosci Methods 196(1):88-98.

Farbman AI, Squinto LM. 1985. Early development of olfactory receptor cell axons. Brain Res 351(2):205-213.

Feron F, Perry C, Cochrane J, Licina P, Nowitzke A, Urquhart S, Geraghty T, Mackay-Sim A. 2005. Autologous olfactory ensheathing cell transplantation in human spinal cord injury. Brain 128(Pt 12):2951-2960.

Gorrie CA, Hayward I, Cameron N, Kailainathan G, Nandapalan N, Sutharsan R, Wang J, Mackay-Sim A, Waite PM. 2010. Effects of human OEC-derived cell transplants in rodent spinal cord contusion injury. Brain Res 1337:8-20.

Graziadei GA, Graziadei PP. 1979a. Neurogenesis and neuron regeneration in the olfactory system of mammals. II. Degeneration and reconstitution of the olfactory sensory neurons after axotomy. J Neurocytol 8(2):197-213.

Graziadei PP, Graziadei GA. 1979b. Neurogenesis and neuron regeneration in the olfactory system of mammals. I. Morphological aspects of differentiation and structural organization of the olfactory sensory neurons. J Neurocytol 8(1):1-18.

Graziadei PP, Levine RR, Graziadei GA. 1978. Regeneration of olfactory axons and synapse formation in the forebrain after bulbectomy in neonatal mice. Proc Natl Acad Sci U S A 75(10):5230-5234. 
Hendricks KR, Kott JN, Gooden MD, Lee ME, Evers SM, Goheen BL, Westrum LE. 1994. Recovery of olfactory behavior. II. Neonatal olfactory bulb transplants enhance the rate of behavioral recovery. Brain Res 648(1):135-147.

Iwai N, Zhou Z, Roop DR, Behringer RR. 2008. Horizontal basal cells are multipotent progenitors in normal and injured adult olfactory epithelium. Stem Cells 26(5):12981306.

Jang W, Youngentob SL, Schwob JE. 2003. Globose basal cells are required for reconstitution of olfactory epithelium after methyl bromide lesion. J Comp Neurol 460(1):123-140.

Lankford KL, Sasaki M, Radtke C, Kocsis JD. 2008. Olfactory ensheathing cells exhibit unique migratory, phagocytic, and myelinating properties in the $\mathrm{X}$-irradiated spinal cord not shared by Schwann cells. Glia 56(15):1664-1678.

Leung CT, Coulombe PA, Reed RR. 2007. Contribution of olfactory neural stem cells to tissue maintenance and regeneration. Nat Neurosci 10(6):720-726.

Li Y, Decherchi P, Raisman G. 2003. Transplantation of olfactory ensheathing cells into spinal cord lesions restores breathing and climbing. J Neurosci 23(3):727-731.

Li Y, Field PM, Raisman G. 1997. Repair of adult rat corticospinal tract by transplants of olfactory ensheathing cells. Science 277(5334):2000-2002.

Li Y, Field PM, Raisman G. 2005. Olfactory ensheathing cells and olfactory nerve fibroblasts maintain continuous open channels for regrowth of olfactory nerve fibres. Glia 52(3):245-251.

Lu J, Feron F, Ho SM, Mackay-Sim A, Waite PM. 2001. Transplantation of nasal olfactory tissue promotes partial recovery in paraplegic adult rats. Brain Res 889(1-2):344-357.

Mackay-Sim A, Feron F, Cochrane J, Bassingthwaighte L, Bayliss C, Davies W, Fronek P, Gray C, Kerr G, Licina P and others. 2008. Autologous olfactory ensheathing cell transplantation in human paraplegia: a 3-year clinical trial. Brain 131(Pt 9):23762386.

Mackay-Sim A, Kittel P. 1991a. Cell dynamics in the adult mouse olfactory epithelium: a quantitative autoradiographic study. J Neurosci 11(4):979-984.

Mackay-Sim A, Kittel PW. 1991b. On the Life Span of Olfactory Receptor Neurons. Eur J Neurosci 3(3):209-215.

Murdoch B, Roskams AJ. 2008. A novel embryonic nestin-expressing radial glia-like progenitor gives rise to zonally restricted olfactory and vomeronasal neurons. J Neurosci 28(16):4271-4282.

Ramon-Cueto A, Nieto-Sampedro M. 1994. Regeneration into the spinal cord of transected dorsal root axons is promoted by ensheathing glia transplants. Exp Neurol 127(2):232-244.

Ramon-Cueto A, Plant GW, Avila J, Bunge MB. 1998. Long-distance axonal regeneration in the transected adult rat spinal cord is promoted by olfactory ensheathing glia transplants. J Neurosci 18(10):3803-3815.

Resnick DK, Cechvala CF, Yan Y, Witwer BP, Sun D, Zhang S. 2003. Adult olfactory ensheathing cell transplantation for acute spinal cord injury. J Neurotrauma 20(3):279-285.

Williams SK, Gilbey T, Barnett SC. 2004. Immunohistochemical studies of the cellular changes in the peripheral olfactory system after zinc sulfate nasal irrigation. Neurochem Res 29(5):891-901.

Windus LC, Claxton C, Allen CL, Key B, St John JA. 2007. Motile membrane protrusions regulate cell-cell adhesion and migration of olfactory ensheathing glia. Glia 55(16):1708-1719. 
Windus LC, Lineburg KE, Scott SE, Claxton C, Mackay-Sim A, Key B, St John JA. 2010. Lamellipodia mediate the heterogeneity of central olfactory ensheathing cell interactions. Cell Mol Life Sci 67(10):1735-1750. 


\section{Figure legends}

Figure 1. OECs proliferate after unilateral bulbectomy. Panels are coronal sections through the olfactory region of postnatal OMP-ZsGreen x S100ß-DsRed mice that had undergone unilateral bulbectomy and then labelled with EdU $4 \mathrm{~h}$ prior to harvest. Primary olfactory neurons are green; OECs are red; EdU labelling is yellow. (A) Two days after bulbectomy, the olfactory epithelium on the bulbectomy side (left nasal cavity, NC-1) was thinner. Cells that had proliferated and labelled with EdU (yellow) were detected in the nasal septum (S), olfactory mucosa (OM) and the bulbar cavity (cavity). Boxed areas are shown in B-E. (B-C) A higher magnification view of the boxed area in the olfactory mucosa of the operated side of a bulbectomised mouse shows EdU positive cells (yellow) in the olfactory epithelium (OE) and lamina propria (LP). Dotted line depicts border between OE and LP. OECs (red) that had proliferated and labelled with EdU (arrow) were identified in the lamina propria close to OECs that were not labelled by EdU (arrowhead). Panel C shows the EdU-only labelling that is depicted in panel B. (D-E) Higher magnification of the boxed area in the bulbectomised cavity showing OECs (red) and EdU labelled cells (yellow). Some OECs were labelled by EdU (arrow) while others were not (arrowhead). Scale bar is $380 \mu \mathrm{m}$ in A; $50 \mu \mathrm{m}$ in B-E.

Figure 2. OECs that had proliferated were detected in the olfactory mucosa and olfactory bulb. Coronal sections through the olfactory mucosa (A-E) and through the bulbar cavity (FJ) five days after bulbectomy showing DsRed positive OECs (red), EdU labelling (yellow), anti-BLBP immunostaining (magenta in C), anti-S100 immunostaining (magenta in $\mathrm{H}$ ) and DAPI (blue). OECs that had proliferated and were labelled by EdU are indicated by arrows, OECs that had not proliferated are indicated by arrowheads. (K) Macrophages labelled with anti-Iba1 antibodies (green). Some macrophages expressed DsRed (arrows with tails); others 
did not (arrowheads). (L) Macrophages (arrows with tails) expressed high levels of DsRed and their rounded morphology and multiple short processes made them readily identifiable. In contrast OECs diffusely expressed DsRed and had extensive membranes (arrows). NC, nasal cavity; OE, olfactory epithelium; LP, lamina propria; S, septum. Scale bar is $50 \mu \mathrm{m}$ in A-K; $25 \mu \mathrm{m}$ in L.

Figure 3. OECs in the lamina propria proliferated after bulbectomy. Panels are coronal sections through the olfactory mucosa, with the nasal cavity to the top; the basal layer of the olfactory epithelium (OE) is indicated by a dashed line in A,D,G. The inset image in panel A shows the location of the images on the operated side. Triple label images with OECs (red), EdU (yellow), and DAPI (blue) are shown in A,D,G with single fluorescent labels of OECs and EdU shown in the panels to the right. EdU was administered $4 \mathrm{~h}$ prior to harvest. Five days after bulbectomy, numerous OECs (red) within the lamina propria (LP) were co-labelled with EdU (yellow, arrows) on the operated (A-C) and contralateral unoperated sides (D-F) of unilaterally bulbectomised mice. In unoperated control animals (G-I) OECs that had proliferated were also present (arrows). A few macrophages with their distinct morphology and intense DsRed expression were scattered throughout the tissue (arrowheads in H). Scale bar is $50 \mu \mathrm{m}$.

Figure 4. OECs in the nerve bundles passing through the cribriform plate proliferated after bulbectomy. Panels are coronal sections through the cribriform plate of unilaterally bulbectomised animals at 5 days after surgery; EdU was administered $4 \mathrm{~h}$ prior to harvest. (A) The olfactory nerve bundles (boxed areas) passing through the cribriform plate contained EdU-labelled OECs. (B-J) Triple label images with OECs (red), EdU (yellow), and DAPI (blue) are shown in B,E,H with single fluorescent labels of OECs and EdU shown in the 
panels to the right. Higher magnification views of the nerve bundles on the operated side (BD) and on the contralateral unoperated side of the same animal (E-G) and in unoperated control animals (H-J). OECs (red) that were co-labelled with EdU (yellow) are indicated by arrows. Scale bar is $185 \mu \mathrm{m}$ in A, $50 \mu \mathrm{m}$ in B-J.

Figure 5. OECs in the olfactory bulbar cavity proliferated after bulbectomy. Panels are coronal sections through the nerve fibre layer, or equivalent region in bulbectomised mice, two days after surgery with EdU administered $4 \mathrm{~h}$ prior to harvest. The inset image in panel A shows the location of the images on the operated side. Triple label images with OECs (red), EdU (yellow), and DAPI (blue) are shown in A,D,G with single fluorescent labels of OECs and EdU shown in the panels to the right. (A-C) The bulbectomised cavity on the operated side, (D-F) the nerve fibre layer on the contralateral unoperated side of the same animal, (GI) the nerve fibre layer of unoperated control animals. OECs (red) that were co-labelled with EdU (yellow) are indicated by arrows. Scale bar is $50 \mu \mathrm{m}$.

Figure 6. The proliferation of OECs increased after unilateral bulbectomy. Quantification of the percentage of OECs that were labelled by EdU up to 14 days after bulbectomy in (A) the lamina propria, (B) the cribriform plate and (C) the olfactory bulbar cavity, with graphs showing data for the ipsilateral operated side and contralateral unoperated side of unilaterally bulbectomised animals and for unoperated control animals. EdU was administered and the animals harvested $4 \mathrm{~h}$ later. Significance was first determined by two-way ANOVA ( $\mathrm{p}<0.0001$ between treatments); then by post-hoc Tukey HSD; * $\mathrm{p}<0.05$ obtained by Tukey HSD is shown for control unoperated animals in comparison to the operated or contralateral unoperated sides. In all graphs, columns show the mean of $n=3$ animals; error bars are the standard deviation of the $n=3$ values. 
Figure 7. OECs migrated from the lamina propria into the bulbar cavity. (A-C) The OECfilled plexus in the operated cavity (dashed line shows extent of cavity) continued to increase in size at least up to 12 days after bulbectomy. Scale bar is $50 \mu \mathrm{m}$. (D-F) Quantification of the percentage of OECs that were labelled by EdU on the ipsilateral operated side and the contralateral unoperated side in (D) the lamina propria, $(\mathrm{E})$ the cribriform plate and $(\mathrm{F})$ the bulbectomised cavity or nerve fibre layer. Significance was first determined by two-way ANOVA $(\mathrm{p}<0.001$ between treatments, $\mathrm{p}<0.001$ between time points); then by post-hoc Tukey test; ** $\mathrm{p}<0.01$ Tukey HSD between time points shown or in comparison to contralateral unoperated side. (G) Quantification of the density of OECs in the cribriform plate after bulbectomy. Significance was first determined by two-way ANOVA $(p<0.05$ between between treatments); then by post-hoc Tukey test; $* \mathrm{p}<0.05$; Tukey HSD in comparison to unoperated controls. In all graphs, columns show the mean of $n=3$ animals; error bars are the standard deviation of the $n=3$ values.

Figure 8. EdU-labelled cells increased in the lamina propria. Panels are coronal sections through the olfactory mucosa at 5-12 days after bulbectomy. Cells that were proliferating at Day 5 were labelled by EdU and the animals harvested at 0-7 days after EdU injection. (A-C) Cells in the basal compartment of olfactory epithelium (OE) were labelled by EdU (arrow in C). By 3 days (D-F) and 7 days (G-I) after EdU labelling, the labelled cells migrated apically from basal layer and differentiated into olfactory receptor neurons (green; arrowhead in F, I). In the lamina propria (LP), an increase in EdU-labelled OECs coincided with the loss of EdU-labelled cells in the basal layer of the olfactory epithelium. Inset image in $\mathrm{H}$ shows higher magnification view of OECs (red) and EdU labelling (white); EdU labelled some 
OECs (arrows) as well as another unidentified cell (arrowhead). Scale bar is $50 \mu \mathrm{m}$ in all panels; $20 \mu \mathrm{m}$ in the inset image in panel $\mathrm{H}$.

Figure 9. (A) Schematic diagram of a sagittal view of the olfactory system with rostral to the right, dorsal to the top. Olfactory sensory axons (blue) project from the epithelium lining the nasal cavity into the olfactory bulb. Olfactory ensheathing cells (red) are in close association with the axons as they project from the lamina propria (LP), through the cribriform plate $(\mathrm{CP})$ and into the nerve fibre layer (NFL). (B) In the pulse-chase experiment, five days after bulbectomy and $4 \mathrm{~h}$ after EdU labelling some OECs in all regions had proliferated (yellow). Within the olfactory mucosa (boxed area), numerous stem cells (grey) that line the basal layer of the olfactory epithelium (OE) were also labelled by EdU (yellow). Seven days later (Day 12), the reduction in cells that had previously proliferated in the stem cell layer coincided with an increase in EdU-positive cells in the neuron layer of the $\mathrm{OE}$ as well as an increase in EdU-positive OECs in the lamina propria (LP) and the cribriform plate (CP). By Day 16, the EdU-labelled OECs had migrated into the bulbectomised cavity. 

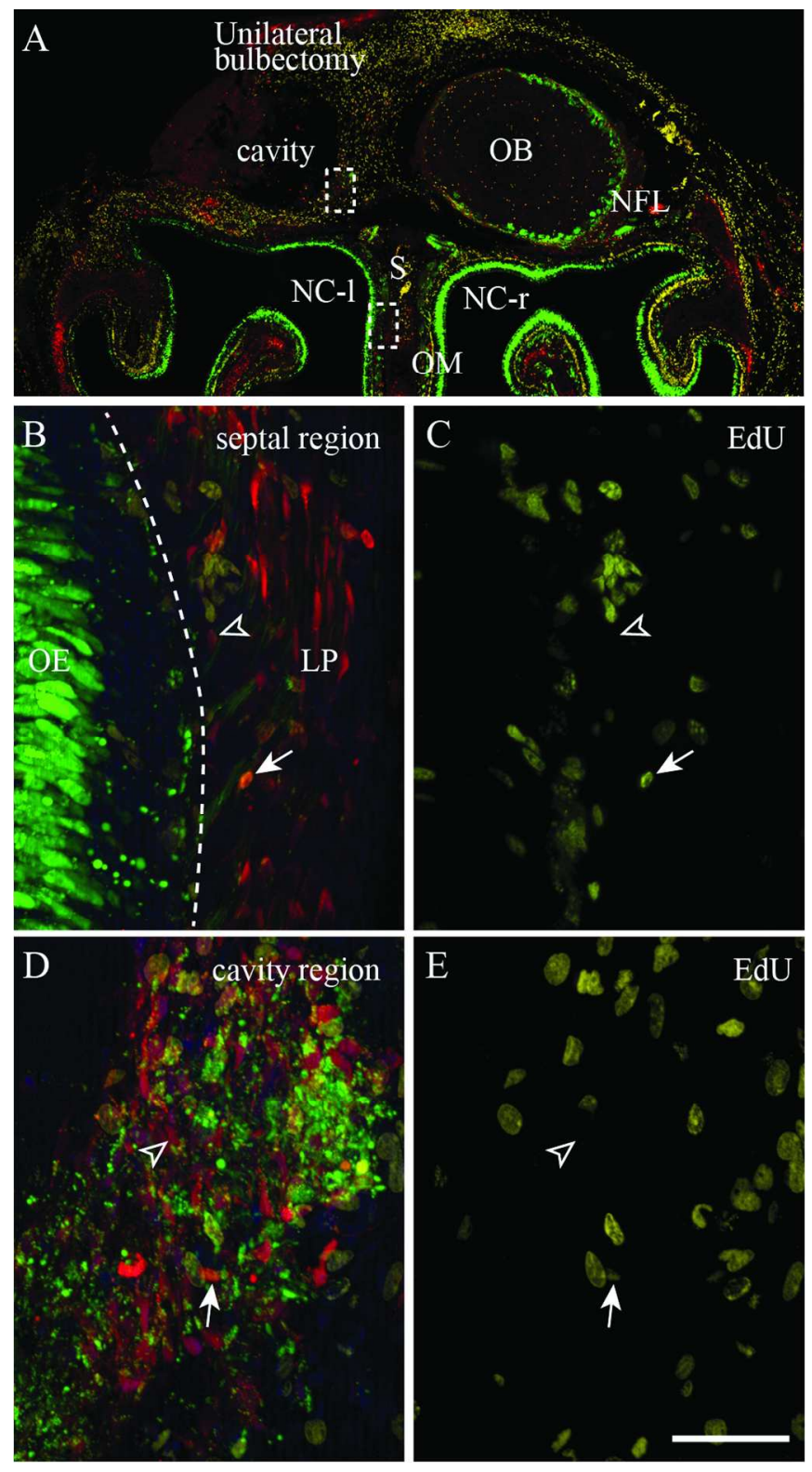

OECs proliferate after unilateral bulbectomy. Panels are coronal sections through the olfactory region of postnatal OMP-ZsGreen x S100ß-DsRed mice that had undergone unilateral bulbectomy and then labelled with EdU $4 \mathrm{~h}$ prior to harvest. Primary olfactory neurons are green; OECs are red; EdU labelling is yellow. (A) Two days after bulbectomy, the olfactory epithelium lining the nasal cavity on the bulbectomy side (left nasal cavity, NC-I) was thinner. Cells that had proliferated were labelled with EdU (yellow) and were detected in the nasal septum (S), olfactory mucosa (OM) and the bulbar cavity (cavity). Boxed areas are shown in B-E. (B-C) A higher magnification view of the boxed area in the olfactory mucosa of the operated side of a bulbectomised mouse shows EdU positive cells (yellow) in the olfactory epithelium (OE) and lamina propria (LP). Dotted line depicts border between OE and LP. OECs (red) that had proliferated and labelled by EdU (arrow) were identified in the lamina propria close to OECs that were not labelled by EdU (arrowhead). Panel $\mathrm{C}$ shows the EdU-only labelling that is depicted in panel B. (D-E) Higher magnification of the boxed area in the bulbectomised cavity showing OECs (red) and EdU labelled cells (yellow). Some OECs were labelled by EdU (arrow) while others were not (arrowhead). Scale bar is $380 \mu \mathrm{m}$ in A; $50 \mu \mathrm{m}$ in B-E. 
$87 \times 159 \mathrm{~mm}(300 \times 300$ DPI $)$

John Wiley \& Sons, Inc. 

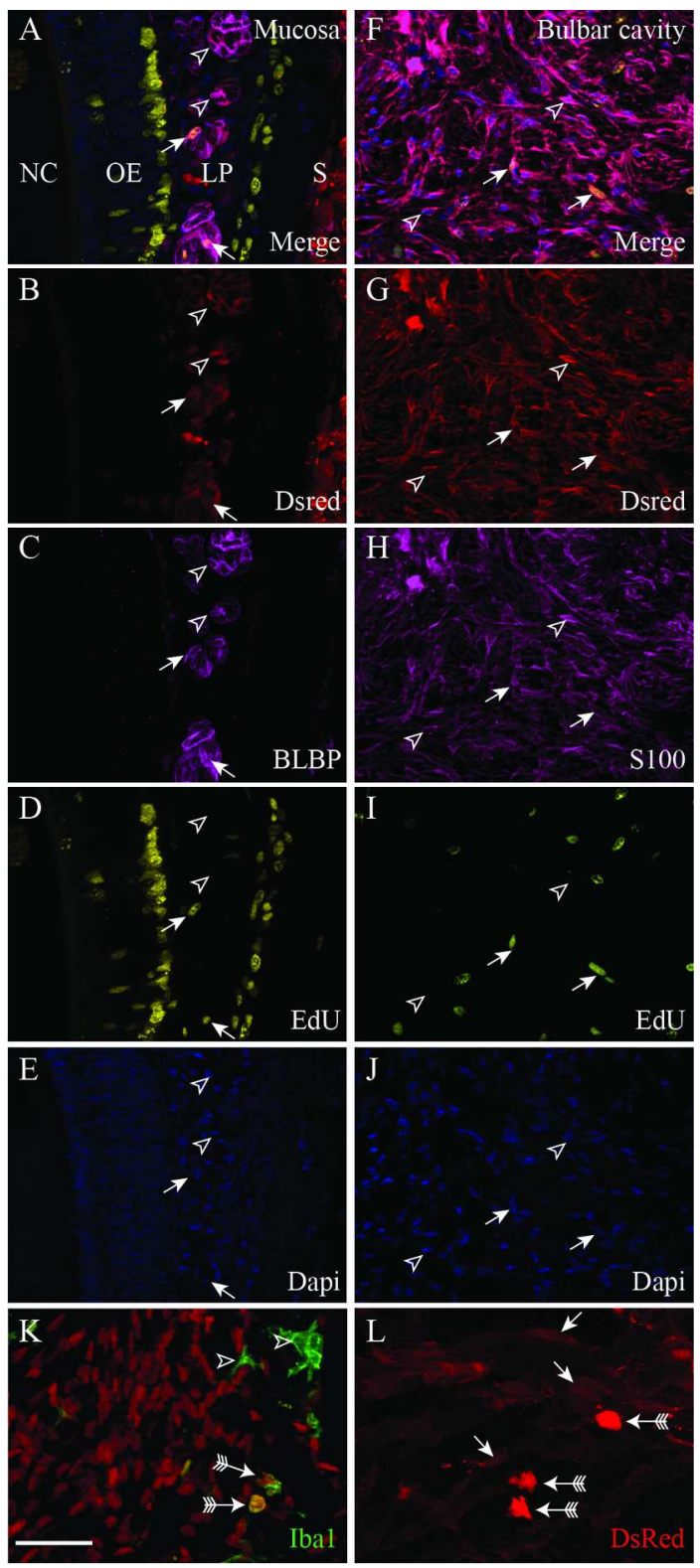

Figure 2. OECs that had proliferated were detected in the olfactory mucosa and olfactory bulb. Coronal sections through the olfactory mucosa (A-E) and through the bulbar cavity (F-J) five days after bulbectomy showing DsRed positive OECs (red), EdU labelling (yellow), anti-BLBP immunostaining (magenta in C), antiS100 immunostaining (magenta in $\mathrm{H}$ ) and DAPI (blue). OECs that had proliferated and were labelled by EdU are indicated by arrows, OECs that had not proliferated are indicated by arrowheads. (K) Macrophages labelled with anti-Iba1 antibodies (green). Some macrophages expressed DsRed (arrows with tails); while others did not (arrowheads). (L) Macrophages (arrows with tails) expressed high levels of DsRed and their rounded morphology and multiple short processes made them readily identifiable. In contrast the numerous OECs diffusely expressed DsRed and had extensive membranes (arrows). NC, nasal cavity; OE, olfactory epithelium; LP, lamina propria; S, septum. Scale bar is $50 \mu \mathrm{m}$ in A-K; $25 \mu \mathrm{m}$ in L.

$88 \times 198 \mathrm{~mm}(300 \times 300$ DPI $)$ 

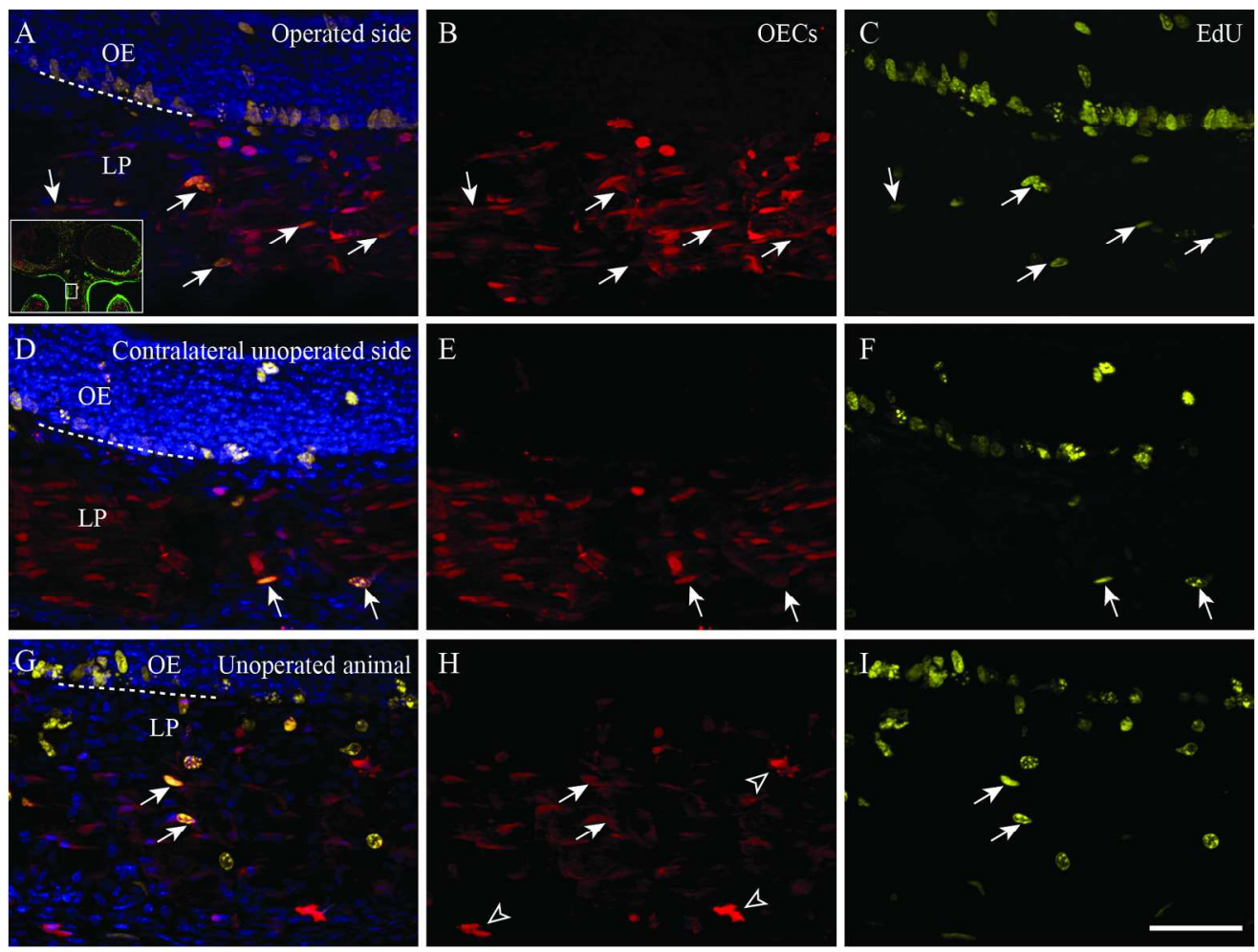

Figure 3. OECs in the lamina propria proliferated after bulbectomy. Panels are coronal sections through the olfactory mucosa, with the nasal cavity to the top; the basal layer of the olfactory epithelium (OE) is indicated by a dashed line in $A, D, G$. The inset image in panel A shows the location of the images on the operated side. Triple label images with OECs (red), EdU (yellow), and DAPI (blue) are shown in A,D,G with single fluorescent labels of OECs and EdU shown in the panels to the right. EdU was administered $4 \mathrm{~h}$ prior to harvest. Five days after bulbectomy, numerous OECs (red) within the lamina propria (LP) were colabelled with EdU (yellow, arrows) on the operated (A-C) and contralateral unoperated sides (D-F) of unilaterally bulbectomised mice. In unoperated control animals (G-I) OECs that had proliferated were also present (arrows). A few macrophages with their distinct morphology and intense DsRed expression were scattered throughout the tissue (arrowheads in $\mathrm{H}$ ). Scale bar is $50 \mu \mathrm{m}$.

$172 \times 130 \mathrm{~mm}(300 \times 300 \mathrm{DPI})$ 

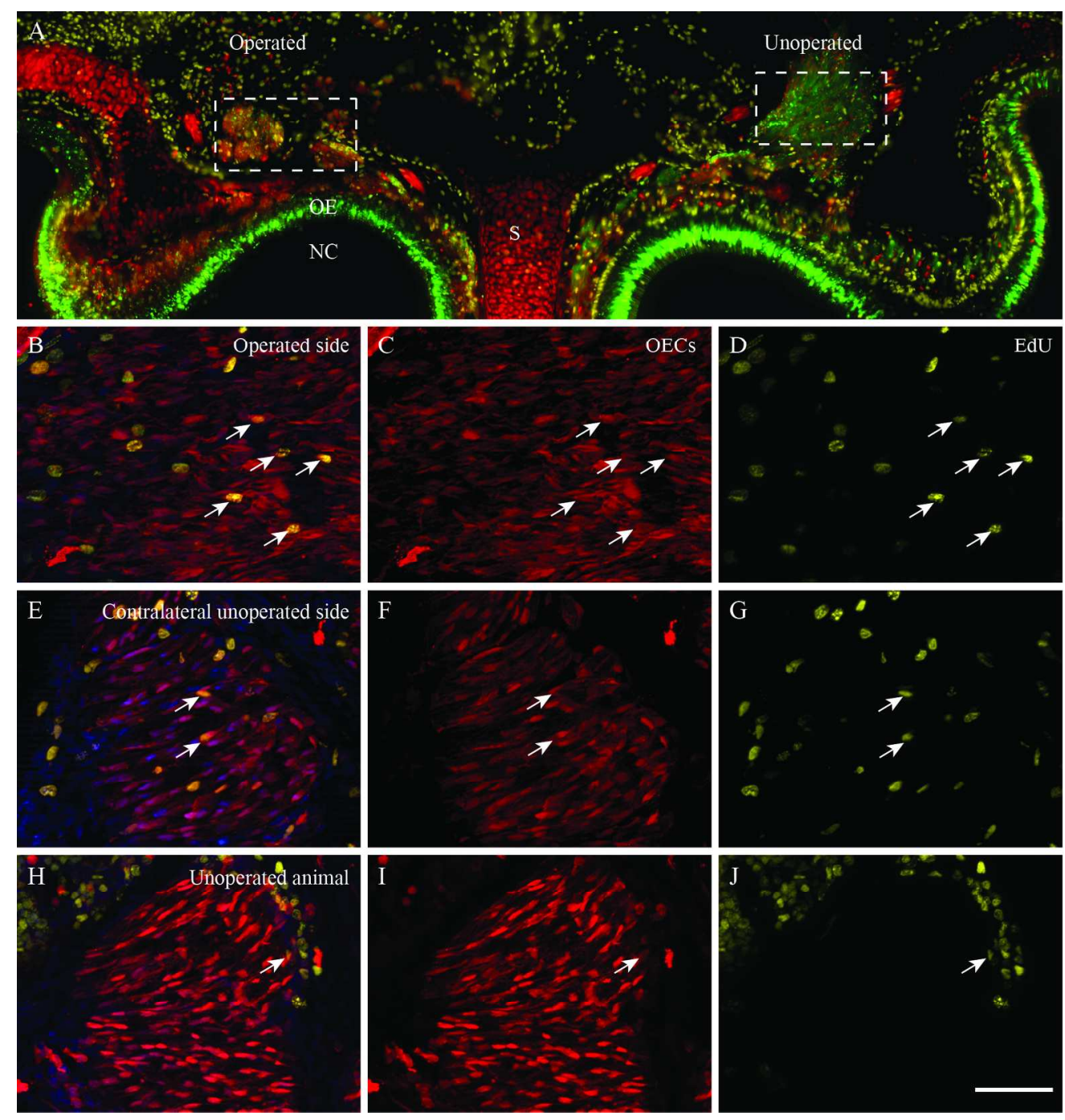

Figure 4. OECs in the nerve bundles passing through the cribriform plate proliferated after bulbectomy. Panels are coronal sections through the cribriform plate of unilaterally bulbectomised animals at 5 days after surgery; EdU was administered $4 \mathrm{~h}$ prior to harvest. (A) The large olfactory nerve bundles (boxed areas) passing through the cribriform plate contained EdU-labelled OECs. (B-J) Triple label images with OECs (red), EdU (yellow), and DAPI (blue) are shown in B,E,H with single fluorescent labels of OECs and EdU shown in the panels to the right. Higher magnification views of the nerve bundles on the operated side (B-D) and on the contralateral unoperated side of the same animal $(E-G)$ and in unoperated control animals $(\mathrm{H}-\mathrm{J})$. OECs (red) that were co-labelled with EdU (yellow) are indicated by arrows. Scale bar is $185 \mu \mathrm{m}$ in A, $50 \mu \mathrm{m}$ in BJ.

$175 \times 185 \mathrm{~mm}(300 \times 300 \mathrm{DPI})$ 

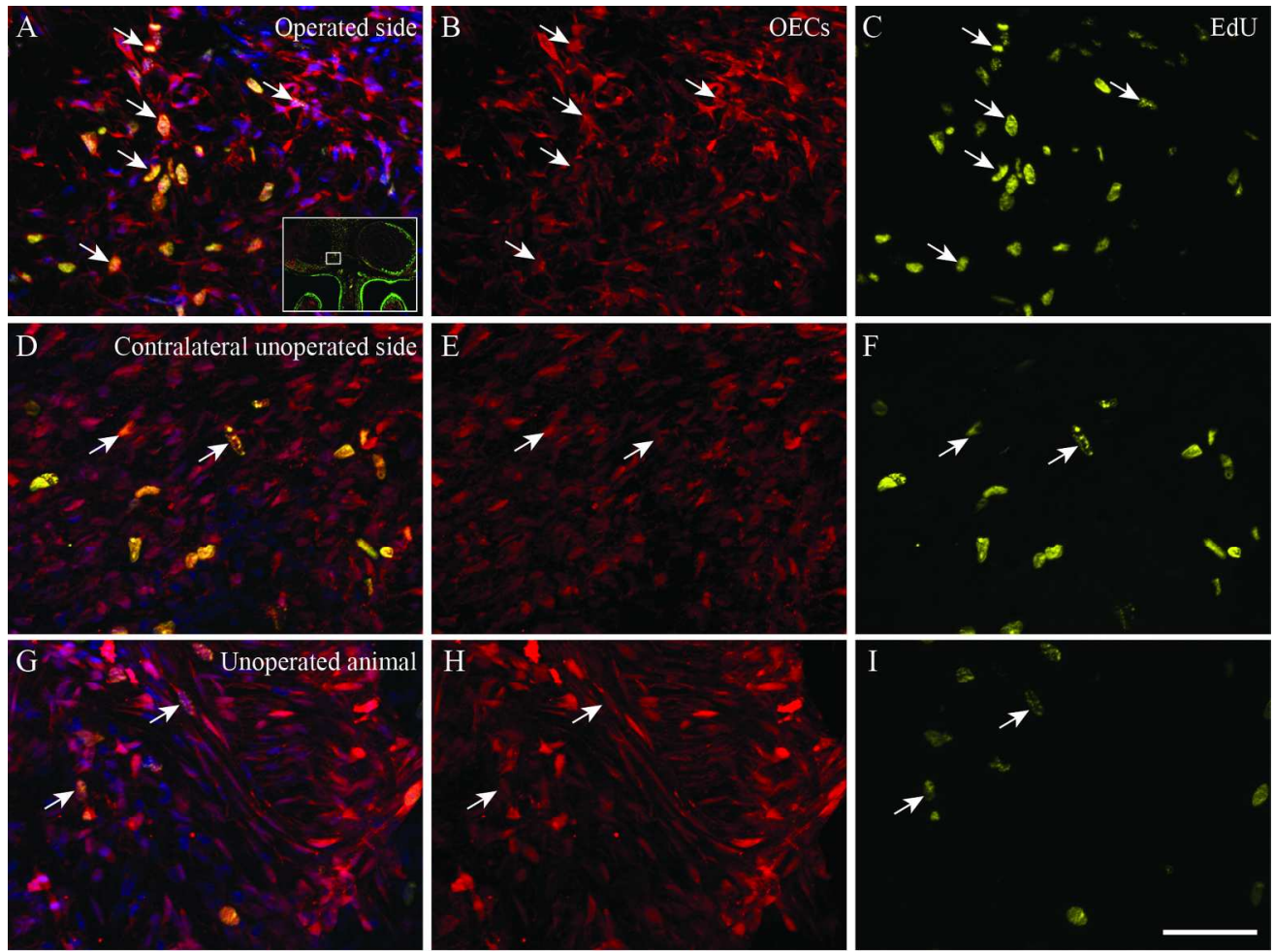

Figure 5. OECs in the olfactory bulbar cavity proliferated after bulbectomy. Panels are coronal sections through the nerve fibre layer, or equivalent region in bulbectomised mice, two days after surgery with EdU administered $4 \mathrm{~h}$ prior to harvest. The inset image in panel A shows the location of the images on the operated side. Triple label images with OECs (red), EdU (yellow), and DAPI (blue) are shown in A,D,G with single fluorescent labels of OECs and EdU shown in the panels to the right. (A-C) The bulbectomised cavity on the operated side, (D-F) the nerve fibre layer on the contralateral unoperated side of the same animal,

(G-I) the nerve fibre layer of unoperated control animals. OECs (red) that were co-labelled with EdU

(yellow) are indicated by arrows. Scale bar is $50 \mu \mathrm{m}$.

$173 \times 129 \mathrm{~mm}(300 \times 300 \mathrm{DPI})$ 


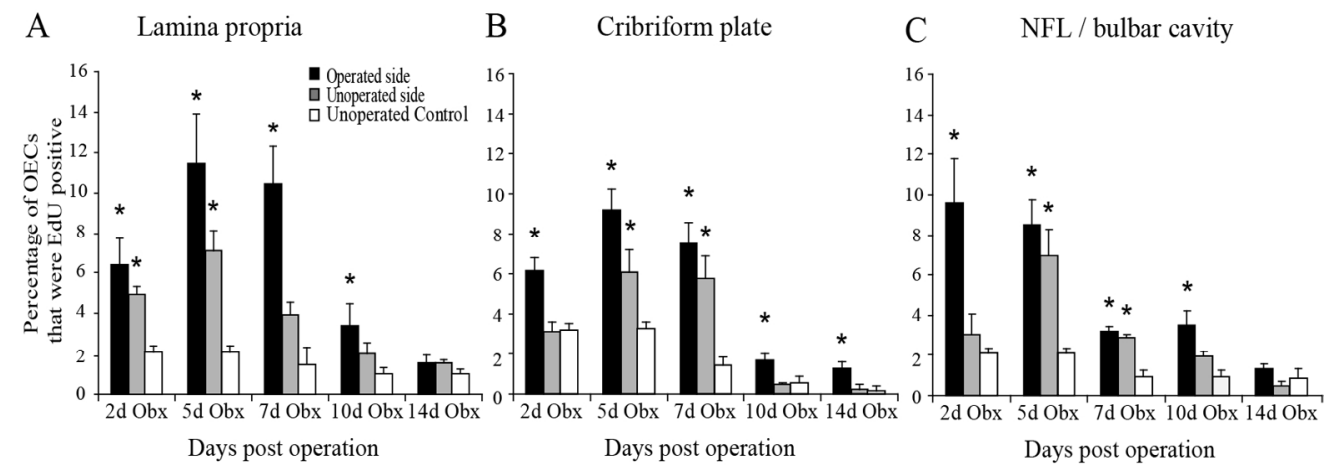

The proliferation of OECs increased after unilateral bulbectomy. Quantification of the percentage of OECs that were labelled by EdU up to 14 days after bulbectomy in $(A)$ the lamina propria, $(B)$ the cribriform plate and $(C)$ the olfactory bulbar cavity, with graphs showing data for the ipsilateral operated side and contralateral unoperated side of unilaterally bulbectomised animals and for unoperated control animals. EdU was administered and the animals harvested $4 \mathrm{~h}$ later. $* \mathrm{p}<0.05$; Tukey HSD.

$145 \times 50 \mathrm{~mm}(300 \times 300$ DPI $)$ 

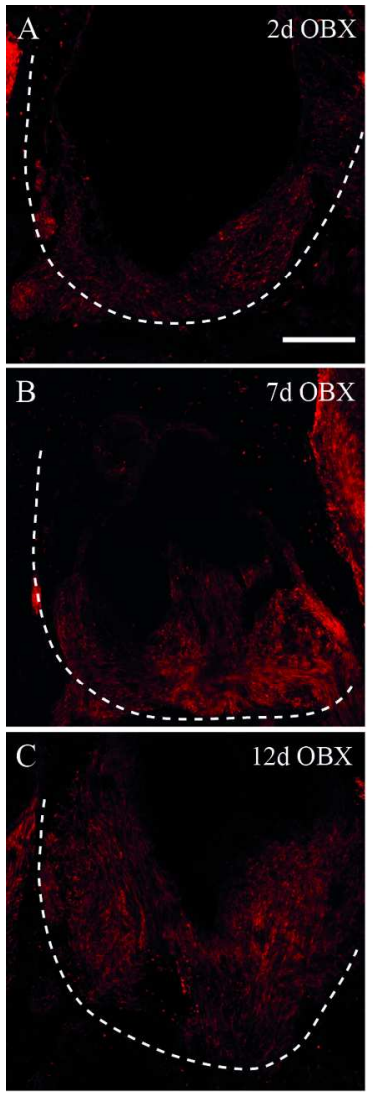

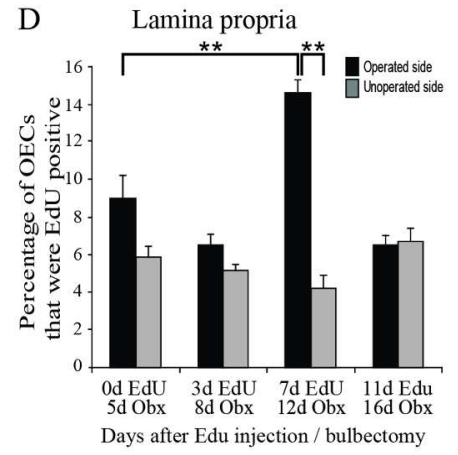

$\mathrm{F}$

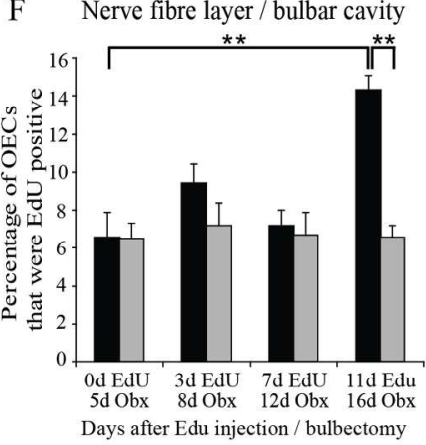

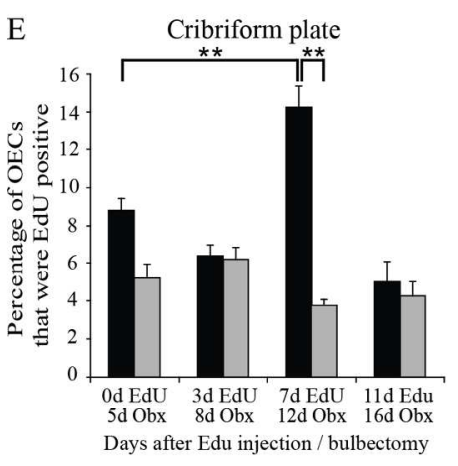

G Density of OECs in CP

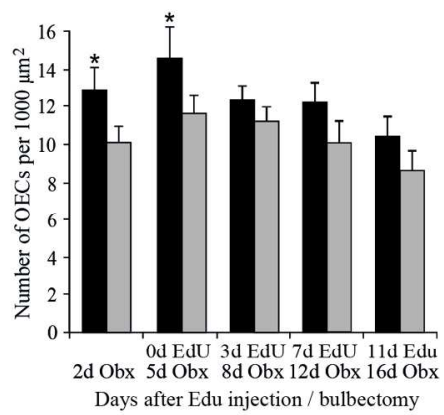

OECs migrated from the lamina propria into the bulbar cavity. (A-C) The OEC-filled plexus in the operated cavity (dashed line shows extent of cavity) continued to increase in size at least up to 12 days after bulbectomy. Scale bar is $50 \mu \mathrm{m}$. (D-F) Quantification of the percentage of OECs that were labelled by EdU on the ipsilateral operated side and the contralateral unoperated side in (D) the lamina propria, $(E)$ the

cribriform plate and $(F)$ the bulbectomised cavity or nerve fibre layer. Significance between the different time points for the operated animals is indicated: ** $p<0.01$; Tukey HSD. (G) Quantification of the density of OECs in the cribriform plate after bulbectomy; significance between operated and unoperated sides at the time points indicated: $p<0.05$; Tukey HSD. $172 \times 143 \mathrm{~mm}(300 \times 300 \mathrm{DPI})$ 

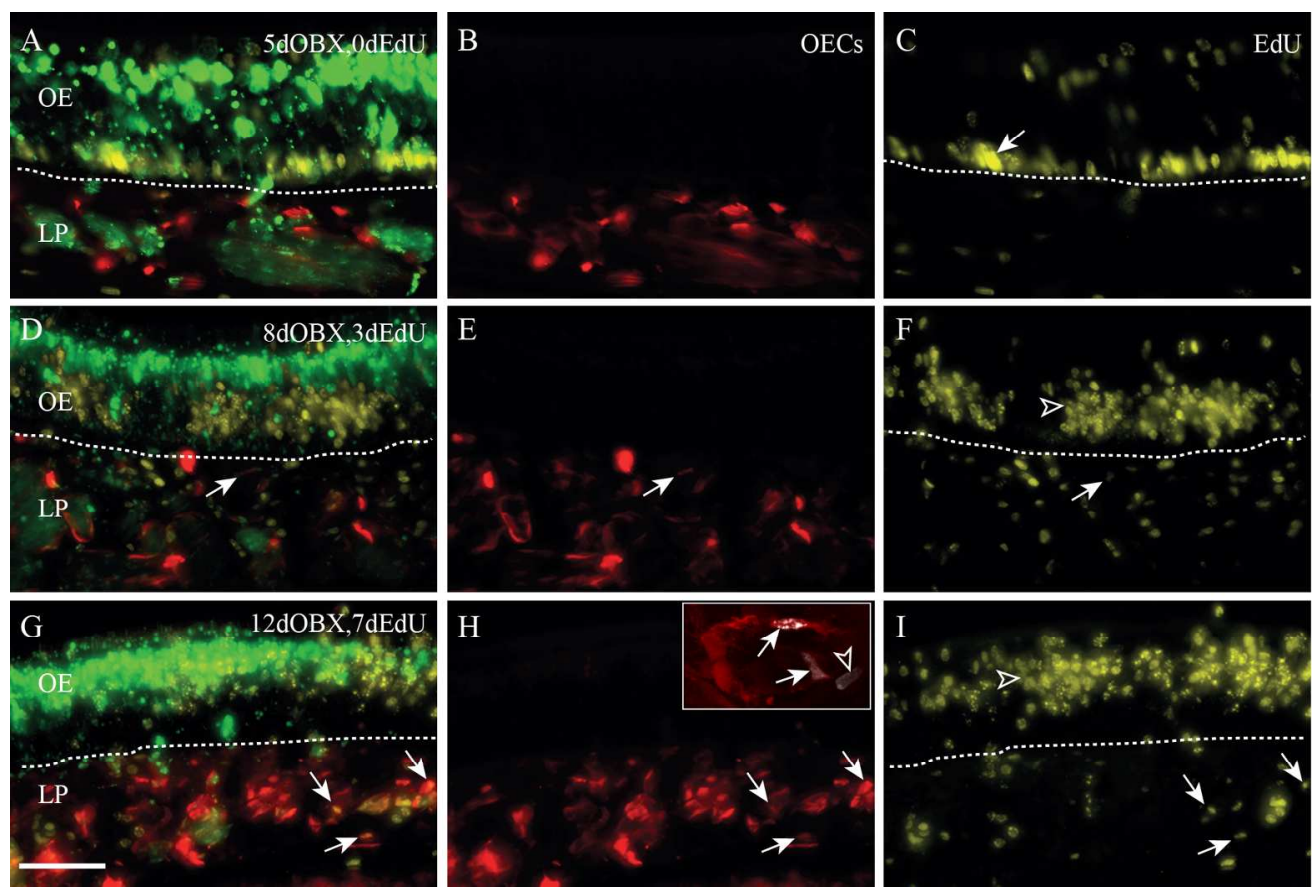

EdU-labelled cells increased in the lamina propria. Panels are coronal sections through the olfactory mucosa at 5-12 days after bulbectomy. Cells that were proliferating at Day 5 were labelled by EdU and the animals harvested at 0-7 days after EdU injection. (A-C) Cells in the basal compartment of olfactory epithelium (OE) were labelled by EdU (arrow in C). By 3 days (D-F) and 7 days (G-I) after EdU labelling, the labelled cells migrated apically from basal layer and differentiated into olfactory receptor neurons (green; arrowhead in $\mathrm{F}$, I). In the lamina propria (LP), an increase in EdU-labelled OECs coincided with the loss of EdU-labelled cells in the basal layer of the olfactory epithelium. Inset image in $\mathrm{H}$ shows higher magnification view of OECs (red) and EdU labelling (white); EdU labelled some OECs (arrows) as well as another unidentified cell (arrowhead). Scale bar is $50 \mu \mathrm{m}$ in all panels; $20 \mu \mathrm{m}$ in the inset image in panel $\mathrm{H}$. $173 \times 116 \mathrm{~mm}(300 \times 300 \mathrm{DPI})$ 


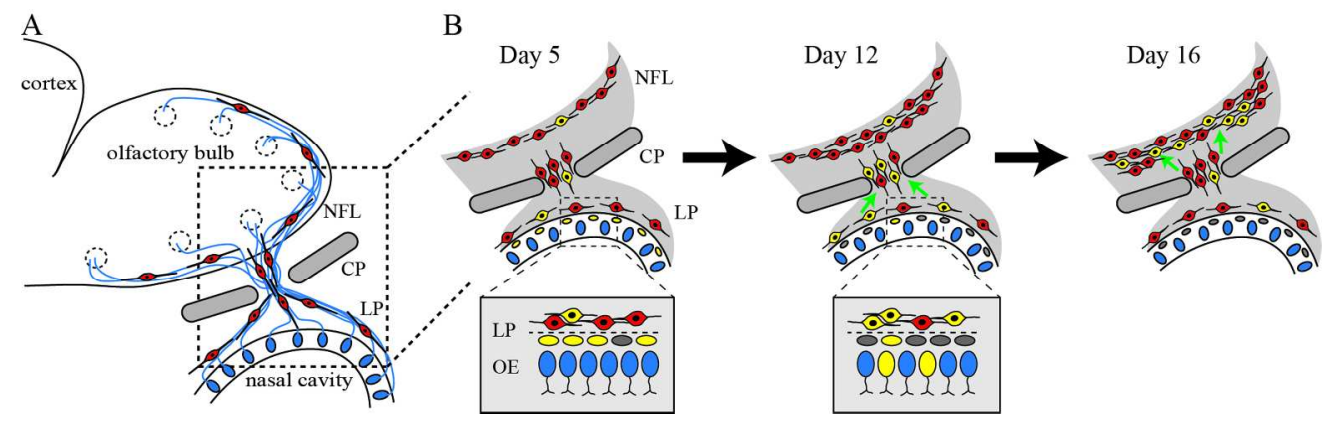

(A) Schematic diagram of a sagittal view of the olfactory system with rostral to the right and dorsal to the top. Olfactory sensory axons (blue) project from the epithelium lining the nasal cavity into the olfactory bulb. Olfactory ensheathing cells (red) are in close association with the axons as they project from the lamina propria (LP), through the cribriform plate (CP) and into the nerve fibre layer (NFL). (B) In the pulsechase experiment, five days after bulbectomy and $4 \mathrm{~h}$ after EdU labelling some OECs in all regions had proliferated (yellow). Within the olfactory mucosa (boxed area), numerous stem cells (grey) that line the basal layer of the olfactory epithelium (OE) were also labelled by EdU (yellow). Seven days later (Day 12), the reduction in cells that had previously proliferated in the stem cell layer coincided with an increase in EdU-positive cells in the neuron layer of the OE as well as an increase in EdU-positive OECs in the lamina propria (LP) and the cribriform plate (CP). By Day 16, the EdU-labelled OECs had migrated into the bulbectomised cavity.

$179 \times 55 \mathrm{~mm}(300 \times 300$ DPI $)$ 